\title{
Premature Termination Codons on Both Alleles of the Type VII Collagen Gene (COL7A1) in Three Brothers with Recessive Dystrophic Epidermolysis Bullosa
}

\author{
Angela M. Christiano, ${ }^{*}$ Yasushi Suga, ${ }^{\ddagger}$ Daniel S. Greenspan, ${ }^{\S}$ Hideoki Ogawa, ${ }^{*}$ and Jouni Uitto *\| \\ *Departments of Dermatology, and "Biochemistry and Molecular Biology, Jefferson Medical College, and Section of Molecular \\ Dermatology, Jefferson Institute of Molecular Medicine, Thomas Jefferson University, Philadelphia, Pennsylvania 19107; ${ }^{\ddagger}$ Department of \\ Dermatology, Juntendo University, Tokyo, Japan; and ${ }^{\S}$ Department of Pathology and Laboratory Medicine, University of Wisconsin, \\ Madison, Wisconsin 53706
}

\begin{abstract}
Epidermolysis bullosa (EB) is a group of heritable mechano-bullous skin diseases classified into three major categories on the basis of the level of tissue separation within the dermal-epidermal basement membrane zone. In the most severe, dystrophic (scarring) forms of EB, blisters form below the cutaneous basement membrane at the level of the anchoring fibrils, which are composed of type VII collagen. Ultrastructural observations of altered anchoring fibrils and genetic linkage to the type VII collagen locus (COL7A1) have implicated COL7A1 as the candidate gene in the dystrophic forms of EB. We have recently cloned the entire cDNA and the gene for human COL7A1. In this study, we describe distinct mutations in both COL7A1 alleles in three brothers with severe, mutilating recessive dystrophic EB (the Hallopeau-Siemens type, HS-RDEB). The patients are compound heterozygotes for two different mutations, both of which result in a premature termination codon in COL7A1, and the parents were shown to be clinically heterozygous carriers of the respective mutations. Premature termination codons in both alleles of COL7A1 appear to be the underlying cause of severe, recessive dystrophic EB in this family. (J. Clin. Invest. 1995. 95:1328-1334.) Key words: genodermatosis - bullous skin diseases - collagen gene mutations $\cdot$ anchoring fibrils
\end{abstract}

\section{Introduction}

Epidermolysis bullosa (EB $)^{1}$ is a group of inherited mechanobullous diseases characterized by easy blistering and fragility of the skin and mucous membranes $(1,2)$. On the basis of clinical, morphologic, and ultrastructural analyses, EB can be

Address correspondence to Jouni Uitto, M.D., Ph.D., Department of Dermatology, Jefferson Medical College, 233 South 10th Street, Rm. 450 BLSB, Philadelphia, PA 19107-5541. Phone: 215-955-5785; FAX: 215-955-5788.

Received for publication 25 April 1994 and in revised form 5 October 1994.

1. Abbreviations used in this paper: COL7A1, the type VII collagen gene; EB, epidermolysis bullosa; PTC, premature termination codons; RDEB, recessive dystrophic epidermolysis bullosa.

J. Clin. Invest.

(C) The American Society for Clinical Investigation, Inc.

0021-9738/95/03/1328/07 \$2.00

Volume 95, March 1995, 1328-1334 divided into three major categories. (a) In the simplex forms, tissue separation occurs at the level of the basal keratinocytes, and mutations in the keratin genes, KRT5 and KRT14, have been shown to underlie this form of $\operatorname{EB}(3,4)$. (b) In the junctional forms of EB, the tissue separation occurs within the cutaneous basement membrane, and the lamina lucida protein laminin 5 (previously known as nicein/kalinin) (5) has been implicated as the candidate gene( $\mathrm{s})$ with subsequent demonstration of mutations in the LAMB3 and LAMC2 genes (6-8). (c) In the dystrophic (scarring) forms of EB, the tissue separation occurs below the basement membrane within the papillary dermis at the level of the anchoring fibrils. Both autosomal dominant and autosomal recessive inheritance patterns have been documented, and the blistering tendency can be highly variable within different forms of EB (1).

The most severe form of recessive dystrophic EB ( the Hallopeau-Siemens type, HS-RDEB), is characterized by mutilating scarring of the hands and feet, joint contractures, strictures of the upper gastrointestinal tract, and the development of aggressive squamous cell carcinomas in affected areas of the skin. These features frequently shorten the life span of the affected individuals to two or three decades. Several lines of evidence suggest that the type VII collagen gene (COL7A1) is the candidate gene in RDEB (9). First, electron microscopy of the skin in affected individuals demonstrates altered morphology and scarcity, or even absence of anchoring fibrils, attachment structures extending from the lamina densa of the cutaneous basement membrane zone to the papillary dermis (10). Previous biochemical studies have indicated that type VII collagen is the major, if not the exclusive, component of the anchoring fibrils (11). The electron microscopic findings are supported by immunofluorescence studies with anti-type VII collagen antibodies, which frequently demonstrate weak, discontinuous, or negative staining reaction for type VII collagen epitopes in the skin of RDEB patients (12). Second, cloning of the type VII collagen gene and identification of informative RFLPs have allowed demonstration of close genetic linkage between the type VII collagen locus, which has been mapped to the p21 region of human chromosome 3 , and both the autosomal dominant and autosomal recessive forms of dystrophic EB (13-16). In particular, genetic linkage analysis of eight families with dominant dystrophic EB, using intragenic or flanking RFLPs, has demonstrated a LOD score $(Z)$ of $>25$ with no recombination (14, $15,17,18)$. Furthermore, examination of 19 informative families with mutilating HS-RDEB with an intragenic PvuII RFLP has demonstrated a LOD score of 3.97 at $\theta=0$ (16). Collectively, these observations suggest that most, if not all, families with the dystrophic forms of EB demonstrate genetic linkage to the locus, with no evidence for locus heterogeneity thus far in COL7A1. 
Type VII collagen is a homotrimer composed of three identical polypeptide subunits, known as $\alpha 1$ (VII) chains (19). Each $\alpha 1$ (VII) chain consists of a central collagenous region, $140 \mathrm{kD}$ in size, flanked by a relatively large amino-terminal noncollagenous (NC-1) domain and a smaller, $20 \mathrm{kD}$, carboxy-terminal noncollagenous (NC-2) domain $(20,21)$. We have recently completed characterization of the intron/exon organization of the type VII collagen gene (22). The gene contains 118 exons in $31 \mathrm{~kb}$ of genomic DNA, more exons than in any previously characterized gene. The primary sequence of NC-1, as deduced from cloned cDNAs, revealed a chimeric arrangement of subdomains with homology to proteins with adhesive properties (20, 23 ). These subdomains include a segment with homology to cartilage matrix protein, nine consecutive fibronectin type IIIlike repeats and a segment with homology to the A domain of von Willebrand factor. During the assembly of anchoring fibrils, two individual type VII collagen molecules are thought to align in an antiparallel orientation stabilized by intermolecular disulfide bonds at the overlapping NC-2 domains. Subsequently, multiple anti-parallel dimer molecules assemble to form anchoring fibrils, which contain the NC-1 domain at each end of these structures.

Cloning of type VII collagen has facilitated molecular analysis of the underlying mutations in different forms of dystrophic EB. For example, we have identified a glycine substitution (G2040S) in the triple helical domain of type VII collagen in a large family with dominant dystrophic EB (DDEB) (24). Furthermore, we identified a single base substitution in two siblings with a mild form of RDEB (mitis type), which resulted in a methionine-to-lysine substitution in a highly conserved segment within the carboxy-terminal noncollagenous (NC-2) domain of type VII collagen (25). In the homozygous state, this missense mutation resulted in a mild scarring form of dystrophic EB, while the mother and a half-brother were heterozygous carriers of the mutation without clinical consequences. In contrast, in patients with severe, mutilating HS-RDEB, we previously identified a homozygous insertion/deletion mutation which leads to premature termination of translation in FNIII domain 4A (26), and more recently, additional mutations in COL7A1 also resulting in premature termination codons (PTCs) $(27,28)$. In three different HS-RDEB patients, the mutations on both alleles lead to premature termination codons, predicting termination of translation of type VII collagen polypeptides.

In this study, we analyzed COL7A1 for mutations underlying HS-RDEB in three male siblings of Japanese ancestry. The results demonstrate distinct mutations in both COL7A1 alleles resulting in PTCs, while the parents were heterozygous carriers with the respective mutations. Collectively, these findings extend and support the concept that premature termination codon mutations on both COL7A1 alleles are the major underlying cause of severe, mutilating HS-RDEB.

\section{Methods}

PCR amplification and heteroduplex analyses. DNA isolated from peripheral blood lymphocytes was used as a template for amplification of genomic sequences within COL7A1. For this purpose, oligonucleotide primers were synthesized on the basis of intronic sequences (Christiano et al., manuscript in preparation) to generate products spanning several exons. To amplify the 674-bp fragment containing exons 7-9, the prim- ers were: upstream primer, 5'GGCTGGGCAAGATAAAGTGA3'; downstream primer, 5'CGTCCCAGCCTGAAGAAAGT3'. The following primers were used to amplify a 760-bp segment containing exons 69-71: upstream primer, 5'TGAGTGCGGATGTTGGGTAG3' ; downstream primer, 5 'AGCAAGAGGTCAGAGGAGCG3'. For PCR amplification, $\sim 500 \mathrm{ng}$ of genomic DNA was used as template, and the amplification conditions were $94^{\circ} \mathrm{C}$ for $7 \mathrm{~min}$; followed by $94^{\circ} \mathrm{C}$ for 45 $\mathrm{s} ; 55^{\circ} \mathrm{C}$ for $45 \mathrm{~s} ; 72^{\circ} \mathrm{C}$ for $45 \mathrm{~s}$, for 40 cycles; in an OmniGene thermal cycler (Marsh Scientific, Inc., Rochester, NY). Amplification buffer contained $1.5 \mathrm{mM} \mathrm{MgCl}_{2}$, and $2 \mathrm{U}$ of Taq polymerase (GIBCO-BRL), in a total volume of $50 \mu \mathrm{l}$. Aliquots of $5 \mu \mathrm{l}$ were analyzed on $1.0 \%$ agarose gel electrophoresis and $10 \mu \mathrm{l}$ of the sample was prepared for heteroduplex analyses according to the manufacturer's recommendation (MDE, AT Biochemicals, Malvern, PA). Heteroduplexes were visualized by staining with ethidium bromide. If a band of altered mobility was detected in heteroduplex analysis, the PCR product was subcloned into a PCR compatible vector (TA; Invitrogen, San Diego, CA), and sequenced by standard techniques (29).

Verification of the mutations. The putative mutations detected in the PCR products were verified at the genomic level in each case. For this purpose, a search for potential changes in restriction endonuclease sites as a result of the mutation was performed. If an altered restriction enzyme site was detected, DNA in the region of the mutation was amplified by PCR, and the products were cleaved with the restriction enzymes DdeI, AciI, and MspI according to the manufacturer's recommendations (Boehringer-Mannheim, Indianapolis, IN; and New England Biolabs Inc., Beverly, MA).

\section{Results}

\section{Strategy for identification of mutations in COL7AI}

To search for mutations in COL7A1 in patients with RDEB, we developed a scanning strategy which is based on PCR amplification of genomic DNA segments, followed by agarose gel electrophoresis and heteroduplex analysis of the PCR products. Each PCR product spans 2 to 5 exons of the gene, which encompasses a total of 118 exons in its entirety (22, and Christiano et al., manuscript in preparation). Thus, a total of $50 \mathrm{PCR}$ products are required to scan the entire COL7A1 gene. In the event that a band with altered mobility in the heteroduplex analysis is noted, the PCR product is subcloned and subjected to nucleotide sequencing by the dideoxynucleotide termination method (29). Using this strategy, in this study we have identified two distinct mutations in three brothers with RDEB. Both mutations cause a premature termination codon within COL7A1 arising by a different mechanism, and predicting the synthesis of a truncated $\alpha 1$ (VII) chain.

\section{Identification of distinct mutations in both alleles of COL7A1}

Clinical features. The probands were 20-, 16-, and 13-yr-old Japanese brothers with extreme fragility of the skin which had been noted at birth. The skin involvement led to extensive mutilating scarring, loss of nails, and joint contractures (Fig. 1). The patients also had blistering of the mucous membranes in the oral cavity, and esophageal strictures which had caused severe malnutrition and anemia. These complications led to the demise of the oldest brother at the age of 21 , while this manuscript was under review.

Diagnostic light and transmission electron microscopy revealed sub-basal lamina dermal-epidermal separation. Furthermore, no anchoring fibrils were observed in the skin of the patient II-2 (Fig. 2). These findings are consistent with the 

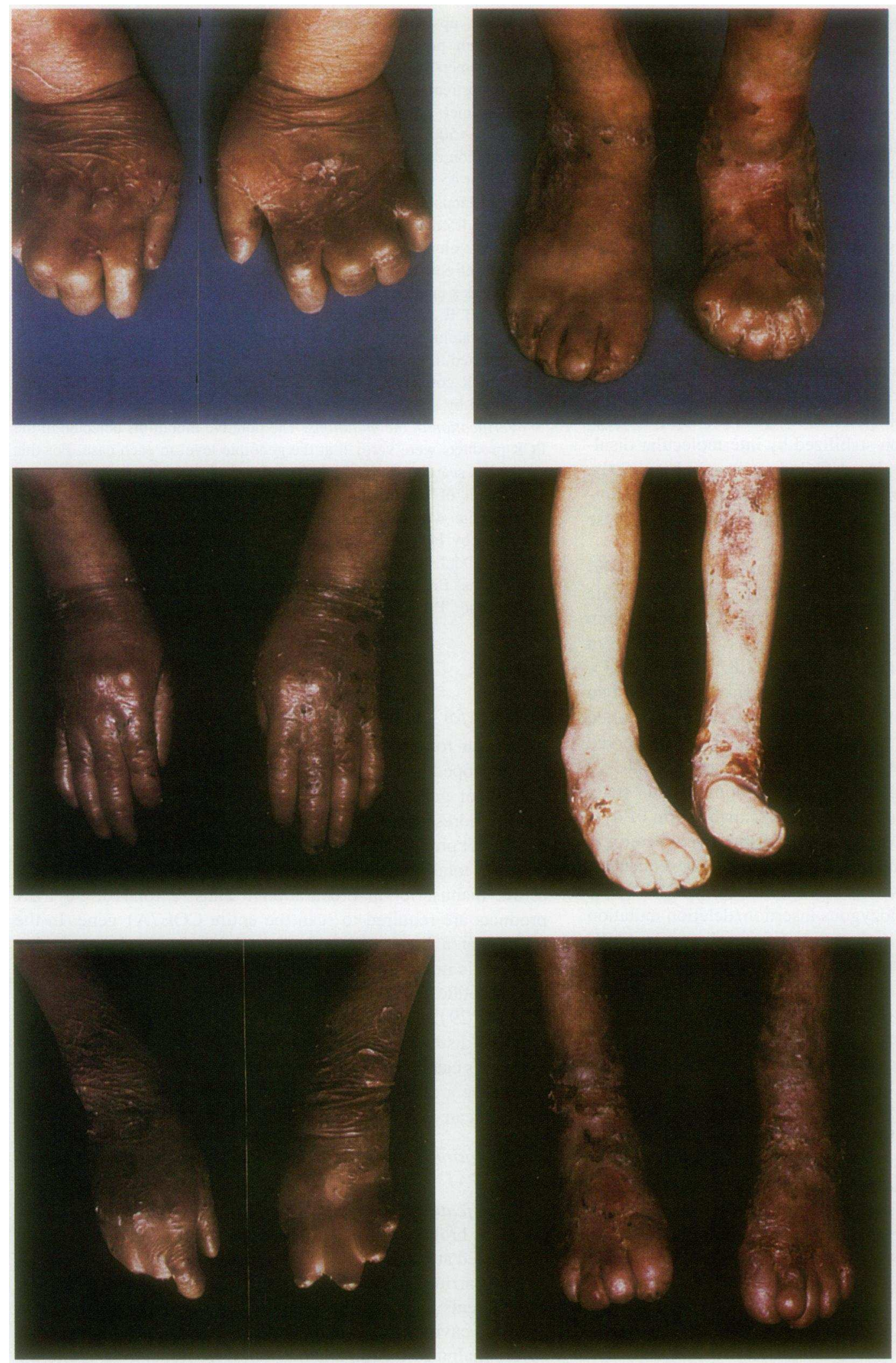

Figure 1. Clinical presentation of the probands affected with the severe, Hallopeau-Siemens type of RDEB. Note the mutilating scarring, fusion of the digits and loss of nails on the hands and feet. The panels from top to bottom represent the 13-, 16-, and 20-yr-old brothers (II-1, II-2, and II3 in Fig. 2), respectively. 


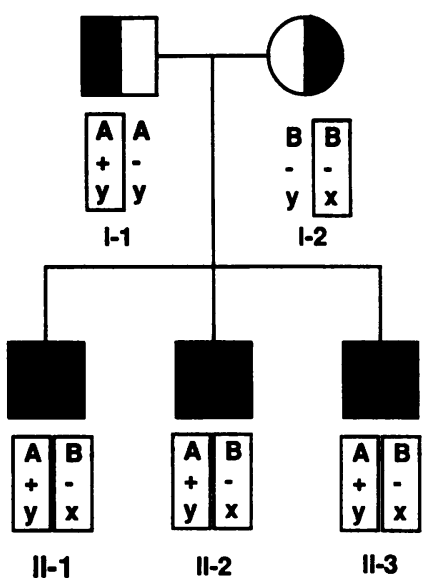

Figure 2. Pedigree of the family. The probands with RDEB (see Fig. 1) are indicated by filled squares. Haplotype analysis beneath each character depicts an intragenic PvuII RFLP in COL7A1 $(A / B)$, the Y $311 \mathrm{X}$ mutation $(y / x)$, and the 5818delC mutation (+/-). The parents are clinically unaffected carriers of one of the mutated alleles (box).

Hallopeau-Siemens type of RDEB (10). The patients had no unaffected siblings, and the parents were clinically normal (Fig. $2)$. There was no evidence of consanguinity.

Identification of mutations. To search for mutations in COL7A1, genomic DNA isolated from peripheral blood was used as the template for PCR amplification, as described above. Putative mutations in two different segments of COL7A1 were identified in the probands. First, a shift in the heteroduplex analysis was noted when the PCR product spanning exons 79 was subjected to analysis (Fig. $3 A$ ). Sequencing of this fragment revealed a C-to-A transversion at position 933 within exon 7 of COL7A1, as compared to the normal allele (Fig. 3 $B$ ). This mutation, which converted a tyrosine codon (TAC) into a nonsense mutation (TAA), is designated Y311X.

The search for the mutation in the other COL7A1 allele of the proband resulted in identification of a shift in heteroduplex analysis when the PCR product spanning exons 69-71 was analyzed (Fig. $4 \mathrm{~A}$ ). Sequencing of the product revealed a deletion of a $\mathrm{C}$ at position 5818 within exon 70 of COL7A1 (Fig. $4 \mathrm{~B}$ ). This deletion, 5818delC, resulted in a frameshift and a premature termination codon 64 amino acids downstream from the site of the nucleotide deletion. The premature termination codon was predicted to result in a truncated $\alpha 1$ (VII) chain which terminates within exon 73 , encoding parts of the noncollagenous "hinge" region within the collagenous domain of the $\alpha 1$ (VII) chain. Thus, the probands in this family are compound heterozygotes with two distinct mutations, each causing a premature termination codon in different regions of COL7A1.

Verification and inheritance of the mutations. To verify these two mutations at the genomic level and to examine the inheritance of the mutations within the family, DNA from the clinically affected siblings and their unaffected parents was analyzed. Heteroduplex analysis by electrophoresis using PCR products spanning exons 7-9, revealed that the mother (individual I-2 in Fig. 2), as well as the probands (II-1, II-2, and II-3) demonstrated a similarly shifted band (Fig. 4, lanes 2-5) while the father's (I-1) did not show such a shift. The mutation, a Cto-A transversion, created a new restriction enzyme site for DdeI (CTAAG). Digestion of amplified DNA with this endonuclease confirmed that the individuals analyzed in lanes 2-5 were heterozygous for the mutation Y311X, while lanes 1 and 6 demonstrated the normal restriction pattern only (Fig. $3 C$ ).

To examine the inheritance of the mutation 5818delC in exon 70 in the family, heteroduplex analysis was performed on the same individuals indicated above. The results revealed that individuals I-1 (the father) as well as II-1, II-2, and II-3 (the probands) demonstrated an altered band, as shown in lanes 1 , and $3-5$ in Fig. $4 \mathrm{~A}$. The mutation $5818 \mathrm{delC}$ in exon 70 resulted in the generation of a new Acil restriction site (GCGG) and the loss of an MspI site (CCGG). Individuals with a band showing altered mobility in the heteroduplex analysis also contained a new restriction enzyme site for the endonuclease Acil,
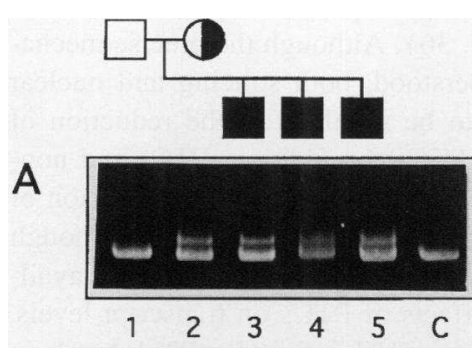

C

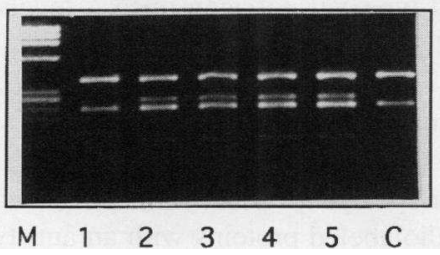

B

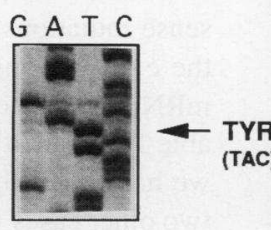

NORMAL

ALLELE

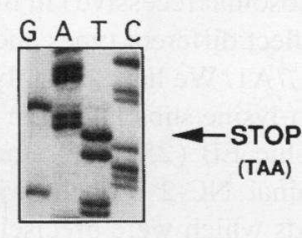

MUTANT

ALLELE Y311X
Figure 3. Heteroduplex analysis of PCR products corresponding to exons 7-9 in COL7A1 in the family. The lane $C$ represents an unrelated healthy control $(A)$. Sequence analysis of the bands demonstrating altered mobility in lanes 2-5 revealed a C-to-A transversion resulting in a nonsense mutation in exon $7(B)$. The nucleotide substitution was verified by DdeI restriction enzyme digestion of the PCR product $(C)$. The mutation creates a new restriction site for this enzyme (CTAAG), and yields digestion fragments of 384 and $221 \mathrm{bp}$ from the normal allele, and 261 and $123 \mathrm{bp}$ from the mutant allele, after cleavage of the 384-bp fragment. The lane $\mathrm{M}$ contains molecular weight markers $(C)$. This mutation converts a tyrosine codon (TAC) to a nonsense mutation (TAA), and is designated $\mathrm{Y} 311 \mathrm{X}$. 


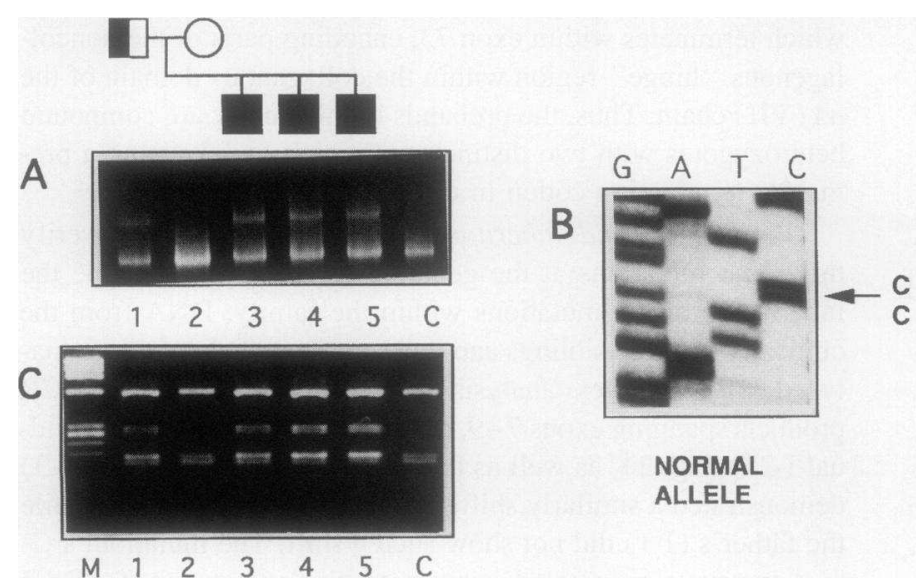

indicating that they were heterozygous for this mutation (Fig. $4 C$ ), and had lost an MspI site (not shown). Thus, the probands with severe HS-RDEB were shown to be compound heterozygotes for both mutations, while their father (I-1) and mother (I-2) were heterozygous carriers of different mutations.

These mutations were not observed in 36 alleles of 18 unrelated unaffected individuals, suggesting that they are not polymorphisms. The mutation Y311X was also not observed in 80 unrelated dystrophic EB patients. However, the mutation 5818delC was observed in the heterozygous state in one unrelated RDEB patient from Japan and his father, suggesting that $5818 \mathrm{delC}$ may be a more common mutation in this gene pool (30).

\section{Discussion}

In this study, we have identified discrete mutations in COL7A1 in both alleles of three brothers with HS-RDEB. Both mutations are predicted to result in the synthesis of truncated $\alpha 1$ (VII) collagen chains. In these patients, a different mutation was identified in each COL7A1 allele, consisting of a nonsense mutation and a deletion of a single $C$ nucleotide, respectively. Each parent of the probands was shown to be a heterozygous carrier of one of the mutations, without clinical consequences.

The spectrum of clinical severity and the mode of inheritance (autosomal dominant vs. autosomal recessive) in the dystrophic forms of EB apparently reflect different types and combinations of mutations within COL7A1. We have recently identified a homozygous methionine-to-lysine substitution in a mild (mitis) form of recessive dystrophic EB (25). This mutation was located in the carboxy-terminal NC-2 domain within a region consisting of 67 amino acids which were precisely conserved between hamster and human COL7A1 sequences (21). This mutation resided 4 and 6 amino acids upstream from two conserved cysteine residues, which have been postulated to participate in intermolecular disulfide bonding during the assembly of anchoring fibrils (19). Thus, this mutation affecting the NC-
Figure 4. Heteroduplex analysis of PCR products containing exons $69-71$ of COL7A1 in the family (A). Sequence analysis of the bands with altered mobility in lanes 1 and 3-5 revealed a one base pair deletion of a $C$ in exon 70 , as shown in $(B)$. The mutation was verified by restriction endonuclease digestion $(C)$. The deletion of one $C$ creates a new restriction enzyme site for Aci I (GCGG) and yields digestion fragments of 539, 187 and $150 \mathrm{bp}$ from the normal allele and 279and 260-bp bands from the mutant allele after digestion of the 539bp band $(C)$. This mutation, designated 5818delC, leads to a frameshift and premature termination codon (TGA) 64 amino acids downstream in exon 73 of COL7A1.
2 domain resulted in a relatively mild clinical phenotype in the homozygous individuals, while the heterozygous carriers were clinically normal.

In cases where premature termination codons have occurred on both COL7A1 alleles in five unrelated pedigrees, including the one described in this study, the consequences have been profound, and the genetic lesions resulted in severe, mutilating scarring due to pronounced fragility of the skin and the mucous membranes. In addition to these families, we have recently identified several different premature termination codon mutations on one COL7A1 allele of unrelated RDEB patients while the mutations in the other COL7A1 allele have not been identified in these cases as yet $(27,28$, and Christiano et al., unpublished observations). Premature termination codons have been described as the underlying cause of several different disorders, with one commonly observed consequence of the mutation. In cystic fibrosis, Marfan syndrome and $\beta$-thalassemia, for example, presence of a PTC or nonsense mutation results in a marked diminution in the mRNA transcript level from the mutant allele (31-34). Previous studies on the effect of nonsense mutations have shown that mRNA stability per se was not affected by the PTC, but that the reduced transcript levels primarily reflect altered RNA processing $(35,36)$. Although the precise mechanisms are not yet well understood, both splicing and nuclear transport of RNAs appear to be involved in the reduction of mRNA transcript levels by PTCs. In addition, PTCs and nonsense mutations have also been shown to result in omission of the exon bearing the PTC from the transcript (37). Although mRNA from the patients examined in this study was not available for evaluation of the effects of PTCs on transcript levels, we have recently examined type VII collagen mRNA levels in two other cases with RDEB. Specifically, in one case with PTCs in both alleles, the type VII collagen mRNA levels were reduced to $14 \%$ of normal. Furthermore, a compound heterozygote with a PTC in one allele and a missense mutation in the other, revealed a reduction in the transcript level to $\sim 12 \%$ of normal. Immunoprecipitation of radiolabeled proteins with an anti-type 
VII collagen antibody yielded no evidence of either intracellular or secreted truncated polypeptides (Christiano et al., unpublished observations). Thus, in the patients (II-1, II-2, and II-3) examined in this study with the most severe, mutilating form of HS-RDEB, PTCs in both alleles of COL7A1 are likely to result in the complete absence of any full-length COL7A1 polypeptides, and consequently, no detectable anchoring fibrils in the skin, as demonstrated by diagnostic transmission electron microscopy.

Precise understanding of the mutations underlying the different forms of EB provides a means for early DNA-based prenatal diagnosis from chorionic villi during the first trimester of pregnancy in families such as this, or by blastomere analysis before implantation $(38,39)$. These DNA-based approaches of prenatal diagnosis will replace the currently used invasive procedure of fetal skin biopsy in families at risk for recurrence of the disease. Furthermore, understanding of the molecular basis of different forms of EB, such as COL7A1 mutations in RDEB, will set the stage for future approaches of gene therapy. Potential possibilities for the gene therapy include stable transfection of autologous keratinocytes with the normal type VII collagen gene, followed by replantation of the transfected keratinocytes expressing the normal gene to denuded areas of the skin $(40,41)$. Alternatively, the use of a biolistic particle accelerator (42) could deliver the normal gene to the stem cell population of the epidermal keratinocytes in the future.

\section{Acknowledgments}

The expert technical assistance of Xin Zhang and Yili Xu is gratefully acknowledged. We appreciate the assistance of Debra Pawlicki and Tamara Alexander in preparing the manuscript. We thank Dr. Arupa Ganguly for stimulating discussions, and the family members themselves for their interest and participation in this study. We appreciate the assistance of the Dystrophic Epidermolysis Bullosa Research Association (D.E.B.R.A.) of America.

This work was supported in part by a grant, PO1-AR38923, from the United States Public Health Service, National Institutes of Health, and by the Dystrophic Epidermolysis Bullosa Research Association of America awarded by the Dermatology Foundation. Dr. Christiano is the recipient of the Society for Investigative Dermatology Research Career Development Award from the Dermatology Foundation.

\section{References}

1. Lin, A. N., and D. M. Carter. 1992. Epidermolysis Bullosa: Basic and Clinical Aspects. Springer-Verlag, New York.

2. Fine, J. D., E. A. Bauer, R. A. Briggaman, D. M. Carter, R. A. Eady, N. B. Esterly, K. A. Holbrook, S. Hurwitz, L. Johnson, A. Lin, R. Pearson, and V P. Sybert. 1991. Revised clinical and laboratory criteria for subtypes of inherited epidermolysis bullosa: a consensus report by the subcommittee on diagnosis and classification of the national epidermolysis bullosa registry. J. Am. Acad. Dermatol. 24:119-153.

3. Epstein, E. H., Jr. 1992. Molecular genetics of epidermolysis bullosa. Science (Wash. DC). 256:799-803.

4. Fuchs, E. 1992. Genetic skin disorders of keratin. J. Invest. Dermatol. 99:671-674.

5. Burgeson, R. E., M. Chiquet, R. Deutzmann, P. Ekblom, J. Engel, H. Kleinman, G. R. Martin, G. Meneguzzi, M. Paulsson, J. Sanes, R. Timpl, K. Tryggvason, Y. Yamada, and P. D. Yurchenco. 1994. A new nomenclature for laminins. Matrix Biol. 14:209-211.

6. Pulkkinen, L., A. M. Christiano, T. Airenne, H. Haakana, K. Tryggvason, and J. Uitto. 1994. Mutations in the $\gamma 2$ chain gene (LAMC2) of kalinin/laminin 5 in the junctional forms of epidermolysis bullosa. Nature Genet. 6:293-298.

7. Aberdam, D., M.-F. Galliano, J. Vailly, L. Pulkkinen, J. Bonifas, A. M. Christiano, K. Tryggvason, J. Uitto, E. H. Epstein, Jr., J.-P. Ortonne, and G. Meneguzzi. 1994. Herlitz's junctional epidermolysis bullosa is genetically linked to mutations in the gene for the $\gamma 2$ subunit (LAMC2) of nicein/kalinin (laminin 5). Nature Genet. 6:299-304.

8. Gerecke, D. R., M.-F. Champliaud, D. W. Wagman, and R. E. Burgeson. 1994. The complete primary structure for a novel laminin chain, the laminin Blk chain. J. Biol. Chem. 269:11073-11080.

9. Uitto, J., and A. M. Christiano. 1992. Molecular genetics of the cutaneous basement membrane zone. Perspective on epidermolysis bullosa and other blistering skin diseases. J. Clin. Invest. 90:687-692.

10. McGrath, J. A., A. Ishida-Yamamoto, A. O'Grady, I. M. Leigh, and R. A. J. Eady. 1993. Structural variations in anchoring fibrils in dystrophic epidermolysis bullosa: correlation with type VII collagen expression. J. Invest. Dermatol. 100:366-372.

11. Sakai, L. Y., D. R. Keene, N. P. Morris, and R. E. Burgeson. 1986. Type VII collagen is a major structural component of anchoring fibrils. J. Cell Biol. 103:1577-1586.

12. Bruckner-Tuderman, L., S. Rüegger, B. Odermatt, Y. Mitsuhashi, and U. W. Schnyder. 1988. Lack of type VII collagen in unaffected skin of patients with severe recessive dystrophic epidermolysis bullosa. Dermatologica. 176:5764.

13. Parente, M. G., L. C. Chung, J. Ryynänen, D. T. Woodley, K. C. Wynn, E. A. Bauer, M.-G. Mattei, M.-L., Chu, and J. Uitto. 1991. Type VII collagen: cDNA cloning and chromosomal mapping of the gene. Proc. Natl. Acad. Sci. USA. 88:6931-6935.

14. Ryynänen, M., R. G. Knowlton, M. G. Parente, L. C. Chung, M.-L. Chu, and J. Uitto. 1991. Human type VII collagen: genetic linkage of the gene (COL7A1) on chromosome 3 to dominant dystrophic epidermolysis bullosa. Am. J. Hum. Genet. 49:797-803.

15. Ryynänen, M., J. Ryynänen, S. Sollberg, R. V. Iozzo, R. G. Knowlton, and J. Uitto. 1992. Genetic linkage of type VII collagen (COL7A1) to dominant dystrophic epidermolysis bullosa in families with abnormal anchoring fibrils. $J$. Clin. Invest. 89:974-980.

16. Hovnanian, A., Duquesnoy, P., Blanchet-Bardon, C., Knowlton, R. G., Amselem, S., Lathrop, M., L. Dubertret, J. Uitto, and M. Goossens. 1992. Genetic linkage of recessive dystrophic epidermolysis bullosa to the type VII collagen gene. J. Clin. Invest. 90:1032-1036.

17. Al-Imara, L., A. J. Richards, R. A. J. Eady, I. M. Leigh, M. Farrall, and F. M. Pope. 1992. Linkage of autosomal dominant dystrophic epidermolysis bullosa in three British families to the marker D3S2 close to the COL7A1. J. Med. Genet. 29:381-382.

18. Gruis, N. A., J. N. B. Bavnick, P. M. Steijlen, J. G. van der Schroeff, A. van Haeringen, R. Happle, E. Mariman, S. E. C. vanBeersum, J. Uitto, B. J. Vermeer, and R. R. Frants. 1992. Genetic linkage between the collagen VII (COL7A1) gene and the autosomal dominant form of dystrophic epidermolysis bullosa in two Dutch kindreds. J. Invest. Dermatol. 99:528-530.

19. Burgeson, R. E. 1993. Type VII collagen, anchoring fibrils, and epidermolysis bullosa. J. Invest. Dermatol. 101:252-255.

20. Christiano, A. M., L. M. Rosenbaum, L. C. Chung-Honet, M. G. Parente, D. T. Woodley, T.-C. Pan, R. Z. Zhang, M.-L. Chu, R. E. Burgeson, and J. Uitto. 1992. The large non-collagenous domain (NC-1) of type VII collagen is amino terminal and chimeric. Homology to cartilage matrix protein, the type III domain of fibronectin and the A domain of von Willebrand factor. Hum. Mol. Genet. 7:475-481.

21. Greenspan, D. S. 1993. The carboxyl-terminal half of type VII collagen, including the non-collagenous NC-2 domain and intron/exon organization of the corresponding region of the COL7Al gene. Hum. Mol. Genet. 2:273-278.

22. Christiano, A. M., G. G. Hoffman, L. C. Chung-Honet, S. Lee, W. Cheng, J. Uitto, and D. S. Greenspan. 1994. Structural organization of the human type VII collagen gene (COL7A1), composed of more exons than any previously characterized gene. Genomics. 21:169-179.

23. Gammon, W. R., M. L. Abernethy, K. M. Padilla, P. S. Prisayanh, M. E. Cook, J. Wright, R. A. Briggaman, and S. W. Hunt. 1992. Noncollagenous (NC1) domain of collagen VII resembles multidomain adhesion proteins involved in tissue-specific organization of extracellular matrix. J. Invest. Dermatol. 99:691696.

24. Christiano, A. M., M. Ryynänen, and J. Uitto. 1994. Dominant dystrophic epidermolysis bullosa: identification of a Gly-to-Ser substitution in the triplehelical domain of type VII collagen. Proc. Natl. Acad. Sci. USA. 91:35549-3553.

25. Christiano, A. M., D. S. Greenspan, G. G. Hoffman, X. Zhang, Y. Tamai, A. N. Lin, H. C. Dietz, A. Hovnanian, and J. Uitto. 1993. A missense mutation in type VII collagen in two affected siblings with recessive dystrophic epidermolysis bullosa. Nature Genet. 4:62-66.

26. Hilal, L., A. Rochat, P. Duquesnoy, C. Blanchet-Bardon, J. Wechsler, N. Martin, A. M. Christiano, Y. Barrandon, J. Uitto, M. Goossens, and A. Hovnanian. 1993. A homozygous frameshift mutation in COL7Al predicts a shortened protein in the generalized mutilating (Hallopeau-Siemens) form of recessive dystrophic epidermolysis bullosa. Nature Genet. 5:287-293.

27. Christiano, A. M., G. Anhalt, S. Gibbons, E. A. Bauer, and J. Uitto. 1994. Premature termination codons in the type VII collagen gene (COL7A1) underlie 
severe, mutilating recessive dystrophic epidermolysis bullosa. Genomics. 21:160168.

28. Hovnanian, A., L. Hilal, C. Blanchet-Bardon, Y. de Prost, A. M. Christiano, J. Uitto, and M. Goossens 1994. Recurrent nonsense mutations within the type VII collagen gene in patients with severe recessive dystrophic epidermolysis bullosa. Am. J. Hum. Genet. 55:289-296.

29. Sanger, F., S. Nicklen, and A. R. Coulson. 1977. DNA sequencing with chain-terminating inhibitors. Proc. Natl. Acad. Sci. USA. 74:5463-5467.

30. Shimizu, H., A. M. Christiano, J. Uitto, A. Tanaka, and T. Nishikawa. 1994. A 1 bp deletion ( 5818 delC) in exon 70 of the type VII collagen gene in a Japanese patient with recessive dystrophic epidermolysis bullosa. International Symposium on Inherited Epidermolysis Bullosa Proceedings. 67 (Abstr.)

31. Baserga, S. J., and E. J. Benz, Jr. 1988. Nonsense mutations in the human $\beta$-globin affect mRNA metabolism. Proc. Natl. Acad. Sci. USA. 85:2056-2060.

32. Hamosh, A., B. C. Trapnell, P. L. Zeitlin, C. Montrose-Rafizadeh, B. J. Rosenstein, R. G. Crystal, and G. R. Cutting. 1991. Severe deficiency of cystic fibrosis transmembrane conductance regulator messenger RNA carrying nonsense mutations R553X and W1316X in respiratory epithelial cells of patients with cystic fibrosis. J. Clin. Invest. 88:1800-1885.

33. Jones, C. T., I. McIntosh, M. Keston, A. Ferguson, and D. J. H. Brock. 1992. Three novel mutations in the cystic fibrosis gene detected by chemical cleavage: analysis of variant splicing and a nonsense mutation. Hum. Molec. Genet. 1:11-17

34. Francomano, C. A. 1993. Four novel FBN1 mutations: significance for mutant transcript level and EGF-like domain calcium binding in the pathogenesis of Marfan syndrome. Genomics. 17:468-475.

35. Urlaub, G., P. J. Mitchell, C. J. Ciudad, and L. A. Chasin. 1989. Nonsense mutations in the dihydrofolate reductase gene affect RNA processing. Mol. Cell. Biol. 9:2868-2880.

36. Dietz, H. C., D. Valle, C. A. Francomano, R. J. Kendzior, Jr., R. E. Pyeritz, and G. R. Cutting. 1993. The skipping of constitutive exons in vivo induced by nonsense mutations. Science (Wash. DC). 259:680-683.

37. McIntosh, I., A. Hamosh, and H. C. Dietz. 1993. Nonsense mutations and diminished mRNA levels. Nature Genet. 4:219.

38. Christiano, A. M., and J. Uitto. 1993. DNA-based prenatal diagnosis of heritable skin diseases. Arch. Dermatol. 129:1455-1459.

39. Yerlinsky, Y., and A. M. Kuliev. 1993. Preimplantation Diagnosis of Genetic Diseases. A New Technique in Assisted Reproduction. Wiley-Liss, New York. 1-55.

40. Jensen, P. K. A., and L. Bolund. 1991. Tissue culture of human epidermal keratinocytes: A differentiating model system for gene testing and somatic gene therapy. J. Cell. Sci. 100:255-259.

41. Carroll, J., E. S. Fenjves, J. A. Garlick, and L. Taichman. 1993. Keratinocytes as a target for gene therapy. In Molecular Biology of the Skin. Academic Press, New York. 269-284.

42. Tang, D.-C., M. DeVit, and S. A. Johnson. 1992. Genetic immunization is a simple method for eliciting an immune response. Nature (Lond.). 356:152154. 